Revue d'histoire de l'Amérique française

74. REVUE D.HISTOIRE DE L'AMÉRIQUE FRANÇAISE

\title{
Cliométrie, économie et histoire : un ménage à trois
}

\section{Ruth Dupré}

Volume 46, numéro 4, printemps 1993

URI : https://id.erudit.org/iderudit/305149ar

DOI : https://doi.org/10.7202/305149ar

Aller au sommaire du numéro

\section{Éditeur(s)}

Institut d'histoire de l'Amérique française

ISSN

0035-2357 (imprimé)

1492-1383 (numérique)

Découvrir la revue

Citer cet article

Dupré, R. (1993). Cliométrie, économie et histoire : un ménage à trois. Revue d'histoire de l'Amérique française, 46(4), 645-663.

https://doi.org/10.7202/305149ar

\section{Résumé de l'article}

Cette note tente de réhabiliter la cliométrie, condamnée sans grand procès par Gilles Paquet dans cette revue. Il est vrai qu'à ses débuts dans les années 1960, c'était la guerre ouverte entre cliomètres et historiens, en bonne partie parce que des travaux comme ceux de Robert Fogel étaient à toutes fins pratiques incompréhensibles pour les non-économistes. Les choses ont bien changé depuis. De plus en plus de cliomètres, comme Douglass North, n'utilisent ni mathématique ni économétrie sophistiquées, abaissant ainsi les barrières interdisciplinaires. Cette note veut informer les historiens de ce qui distingue la cliométrie des autres approches de l'étude de l'histoire et retracer les grandes lignes de son évolution en Amérique du Nord dans les trente dernières années. 


\title{
CLIOMÉTRIE, ÉCONOMIE ET HISTOIRE: UN MÉNAGE À TROIS
}

\author{
RUTH DUPRÉ \\ École des Hautes études commerciales
}

\section{RÉSUMÉ}

Cette note tente de réhabiliter la cliométrie, condamnée sans grand procès par Gilles Paquet dans cette revue. Il est vrai qu'à ses débuts dans les années 1960, c'était la guerre ouverte entre cliomètres et historiens, en bonne partie parce que des travaux comme ceux de Robert Fogel étaient à toutes fins pratiques incompréhensibles pour les non-économistes. Les choses ont bien changé depuis. De plus en plus de cliomètres, comme Douglass North, n'utilisent ni mathématique ni économétrie sophistiquées, abaissant ainsi les barrières interdisciplinaires. Cette note veut informer les historiens de ce qui distingue la cliométrie des autres approches de l'étude de l'histoire et retracer les grandes lignes de son évolution en Amérique du Nord dans les trente dernières années.

\section{ABSTRACT}

This note attempts to rehabilitate cliometrics, condemned rather casually by Gilles Paquet in this review. Undoubtedly, there was at its beginnings in the sixties open warfare between cliometricians and historians, mostly because studies like Robert Fogel's econometric models were practically incomprehensible for noneconomists. The situation has changed enormously since. Many cliometricians, like Douglass North, do not use sophisticate mathematics or econometrics, lowering in this way interdisciplinary barriers. This note wishes to inform historians on what distinguishes cliometrics from other approaches to the study of history and to provide them a brief overview of its evolution in North America in the last thirty years.

Dans son article «Économie et histoire: les liaisons dangereuses», Gilles Paquet règle son compte à la «New Economic History» en la condamnant sans appel. Il n'y consacre qu'une demi-page où il n'est question que d' «opération douloureuse qui va faire plus de mal que de bien», de «ravages», de «divorce», et de «période noire». Cela nous paraît un traitement quelque peu rapide du mouvement qui, qu'on le veuille ou pas, domine la scène de l'histoire économique dans le monde anglo-saxon depuis la fin des années 1960. 
Gilles Paquet traite la cliométrie ${ }^{1}$ comme un bloc monolithique et ignore de plus à quel point elle a changé durant sa trentaine d'années d'existence. On ne peut certes dénier les nombreux excès de ses débuts. L'arrogance et les prétentions des jeunes loups qui sont montés aux barricades étaient grandes. Elles s'attirèrent naturellement de fortes réactions d'hostilité, souvent plus émotionnelles que rationnelles, des praticiens plus traditionalistes de l'histoire économique. La guerre ouverte est cependant chose du passé et a fait place depuis un bon moment à la coexistence pacifique. De très nombreux cliomètres en commençant par les plus célèbres - Robert Fogel, Douglass North, Lance Davis, William Parker, ou Paul David - tentent de faire bon ménage avec leurs collègues économistes et avec les historiens qui le veulent bien. De tels ménages à trois ne seront sans doute jamais des relations de tout repos mais probablement pas aussi dangereuses que Gilles Paquet le croit.

Parce que plusieurs lecteurs de cette revue ne sont sans doute pas familiers avec la cliométrie, il nous a paru important de tenter de la réhabiliter. C'est l'objectif de cette note ${ }^{2}$.

\section{1 - LA CLIOMÉTRIE, CE N'EST PAS LE NOM D'UNE MALADIE}

Il est vrai que plusieurs cliomètres se plaisent à mettre en exergue ce dialogue de Johnson et Boswell:

1. À ses débuts, il était naturel de parler de «nouvelle histoire économique». Titre hautement périssable, il fut vite remplacé par cliométrie et par histoire économétrique, cette dernière dans le titre de deux des principaux ouvrages de la nouvelle école, ceux de A. H. Conrad et J. R. Meyer, The Economics of Slavery and Other Studies in Econometric History, et de R. Fogel, Railroads and American Economic Growth: Essays in Econometric History (tous deux: Chicago, Aldine Publishing, 1964). Cliométrie, un néologisme pour exprimer à la fois le caractère dualiste de l'histoire économique et l'emprise des économistes sur la discipline, est devenue l'appellation la plus utilisée. Depuis quelques années, certains proposent de la remplacer parce qu'ils trouvent le terme rébarbatif si ce n'est barbare pour les non-économistes, par économie historique. Voilà pourquoi l'ouvrage de référence de Donald McCloskey, le plus fervent défenseur de la nouvelle appellation, est intitulé A Bibliography of Historical Economics to 1980 (Cambridge University Press, 1990) et le tout dernier groupe de cliométrie à se former en juillet 1991 a choisi de s'appeler «European Association of Historical Economics». Nous nous en tiendrons dans cette note à cliométrie, le nom encore le plus courant, mais le lecteur devrait garder à l'esprit qu'il peut rencontrer les quatre dans la littérature.

2. La Revue d'histoire de l'Amérique française semble avoir été bien peu touchée par ce mouvement. Je n'ai pas procédé à un examen systématique de la revue mais j'ai feuilleté les index décennaux et plusieurs articles à thème économique. Ironiquement - et les lecteurs et collaborateurs de la revue me corrigeront si je me trompe - l'article le plus dans l'esprit cliométrique est celui de G. Paquet et J.-P. Wallot, «Crise agricole et tensions socio-ethniques dans le Bas-Canada, 1802-1812: éléments pour une réinterprétation", RHAF, 26,2 (septembre 1972): 185-237. Ils utilisent comme appareil analytique un graphique d'offre et de demande et proposent le postulat de la rationalité (limitée à la Simon) pour remplacer celui du conservatisme de la thèse Ouellet. 
Boswell: «Sir Alexander Dick tells me that he remembers having a thousand people in a year to dine at his house.»

Johnson: «That, Sir, is about three a day.»

Boswell: «How your statement lessens the idea.»

Johnson: «That, Sir, is the good of counting. It brings every thing to a certainty, which before floated in the mind indefinitely. ${ }^{3}{ }^{\prime}$

et que de nombreux historiens reprennent la fameuse citation d'Arthur Schlesinger que «almost all important questions are important precisely because they are not susceptible to quantitative answers ${ }^{4} »$. Il n'en demeure pas moins que l'utilisation de statistiques n'est pas propre à l'économiste et n'avait au moment de la révolution cliométrique rien de bien nouveau. Dès 1931, J. H. Clapham, le grand spécialiste de l'histoire économique européenne, n'exhortait-il pas chaque historien à acquérir ce qu'on pourrait appeler un sens statistique, l'habitude de se demander à propos de toute institution, politique, regroupement ou mouvement: quelle est sa taille? sa fréquence? sa représentativité? Inutile d'ajouter que les historiens ont répondu à son appel ${ }^{5}$.

Bien sûr les cliomètres, empruntant leurs techniques à l'économétrie, soumettent souvent leurs données à une analyse statistique plus sophistiquée que celle des historiens ${ }^{6}$. Il faut faire attention toutefois de ne pas confondre cliométrie et économétrie. Plusieurs chercheurs à l'avant-scène de la cliométrie ne font presqu'aucune

3. Boswell, Life of Johnson (Londres, Everyman's Edition, 1949), 2: 456. J'ai retrouvé cette exergue notamment dans P. D. McClelland, Causal Explanation and Model Building in History, Economics, and the New Economic History (Cornell University Press, 1975), 211, et deux fois chez D. McCloskey dans «The Achievements of the Cliometrics School», Journal of Economic History, 38,1 (mars 1978): 17, et dans «The Industrial Revolution 1780-1860: a Survey», J. Mokyr, ed., The Economics of the Industrial Revolution (Rowman \& Allanheld Publ., 1985), 55.

4. Par exemple, G. R. Elton dans R. W. Fogel et G. R. Elton, Which Road to the Past? Two Views of History (Yale University Press, 1983), 100. La citation provient de A. Schlesinger Jr, "The Humanist Looks at Empirical Social Research», American Sociological Review, 27 (décembre 1962): 770.

5. J. H. Clapham, «Economic History as a Discipline», E. R. A. Seligman et A. Johnson, ed., Encyclopedia of the Social Sciences (New York, Macmillan, 1931), 5: 328. Les historiens québécois qu'il m'a été donné de lire ou d'écouter récemment - tels le groupe SOREP, Claude Bellavance, Christian Dessureault, Daniel Massicotte et Yves Otis - manipulent, bien que de manière différente, des quantités aussi impressionnantes de statistiques que les cliomètres.

6. Qu'elles soient plus sophistiquées ne signifie pas qu'elles soient nécessairement plus utiles ou plus pertinentes. 
utilisation de l'économétrie. C'était le cas de deux de ses plus illustres fondateurs, Douglass North et Jonathan Hughes, dans leurs travaux sur le changement institutionnel et l'entrepreneurship. Actuellement, c'est le cas de presque tous les cliomètres, et il y en a plusieurs, intéressés par «l'économie politique» et les institutions?

Statistique et économétrie ne sont donc pas les traits distinctifs de la cliométrie même si on a eu longtemps l'habitude de la définir comme étant l'application de la théorie économique et des méthodes quantitatives à l'étude de l'histoire. Ce qui distingue la cliométrie, c'est l'utilisation de la théorie économique dite néo-classique pour analyser des phénomènes historiques ${ }^{8}$. Or, même sous sa forme la plus élémentaire, sans l'artillerie lourde de la mathématique et de l'économétrie, cette théorie économique est loin d'avoir bonne presse en dehors du cercle des économistes. Ceci pourrait expliquer, en partie du moins, les difficultés de communication entre cliomètres et historiens intéressés à l'histoire économique.

D'abord, comme le montre bien Paquet, le raisonnement déductif de l'économiste et celui plutôt inductif de l'historien sont de fort différents modes de pensée qui pourraient en principe se compléter mais qui le plus souvent s'affrontent. La modélisation choque ou effraie souvent l'historien qui la considère comme une simplification

7. Douglass North a écrit quatre livres sur le changement institutionnel: Institutional Change and American Economic Growth (Cambridge, Cambridge University Press, 1971) avec Lance Davis; The Rise of the Western World: a New Economic History (Cambridge, Cambridge University Press, 1973) avec R. P. Thomas; Structure and Change in Economic History (New York, W. W. Norton, 1981) et Institutions, Institutional Change, and Economic Performance (Cambridge, Cambridge University Press 1990). Jonathan Hughes a analysé le rôle des entrepreneurs dans The Vital Few. American Economic Progress and its Protagonists (Boston, Houghton Miffin Co, 1965; deuxième édition, Oxford University Press, 1986) et celui du gouvernement dans The Governmental Habit (New York, Basic Books, 1977). Peut-être le meilleur exemple de la «nouvelle économie politique» est Gary Libecap. Voir son Contracting for Property Rights (New York, Cambridge University Press, 1990) ou encore «Institutional Choice and the Development of U.S. Agricultural Policies in the 1920s», Journal of Economic History, 51,2 (Juin 1991): 397-410. Pour un sujet qui touche de plus près les lecteurs de la $R H A F$, voir M. B. Percy et R. Szostak, «The Political Economy of the Abolition of Seigneurial Tenure in Canada East», Explorations in Economic History, 29,1 (janvier 1992): 51-68.

8. On retrouve la définition traditionnelle avec quelques variantes dans tous les volumes d'histoire économique américaine et les très nombreux articles et livres qui ont été écrits dans les années 1960 et 1970 pour expliquer, défendre, ou démolir la cliométrie. Souvent à l'avantgarde, Donald McCloskey proposait dès 1978 la définition: «a cliometrician is an economist applying economic theory (usually simple) to historical facts (not always quantitative) in the interest of history (not economics)» dans «The Achievements of the Cliometrics School», 15. Qu'elle soit devenue la définition préférée est confirmé par le récent historique du mouvement écrit par R. Whaples à l'occasion du cinquantenaire de l'Economic History Association, "A Quantitative History of the Journal of Economic History and the Cliometric Revolution", Journal of Economic History, 51,2 (juin 1991): 289-301 (voir en particulier 293-294). 
à outrance, un réductionnisme somme toute peu fructueux parce qu'il impose un caractère artificiellement simple à une réalité trop riche et complexe. Pour le cliomètre, au contraire, la modélisation est essentielle afin d'y voir clair: c'est la lampe de poche dans les ténèbres de la réalité historique.

Peut-être encore plus choquant que la méthode déductive est le postulat de la rationalité économique selon lequel l'homo æconomicus, mû par son intérêt personnel, maximise sous contrainte son utilité ou ses profits. Cette vision de l'être humain prête facilement à la caricature et les «anti-utilitaristes» ne s'en privent pas: où, demandent-ils, a-t-on déjà vu un monde composé d'êtres totalement et en tout temps rationnels et calculateurs? Il n'est heureusement pas besoin d'aller jusque-là. La théorie économique néo-classique est simplement une théorie des choix et la maximisation sous contrainte un outil d'analyse qui n'implique rien de plus que les individus tentent, du mieux qu'ils le peuvent, de tenir compte des bénéfices et des coûts de leurs actions. Un comportement qui caractérise sûrement la grande majorité des êtres humains maintenant comme avant. C'est l'hypothèse sur laquelle repose, et qui de fait distingue, la logique économique. Voilà à quoi depuis Adam Smith, qui écrivait $\mathrm{La}$ richesse des nations en 1776, on reconnaît un économiste et par le fait même, un cliomètre.

Or, ce postulat de la rationalité économique provoque assez souvent des réactions quasi viscérales chez les non-économistes. À plus forte raison si l'on tente de l'appliquer à un contexte qui ne correspond pas à une économie de marché moderne comme l'ont fait, par exemple, Douglass North et R. P. Thomas dans The Rise of the Western World ou encore, plus près de nous, Robert Armstrong dans Structure and Change: an Economic History of Quebec. On les accuse alors, à tort il me semble, de considérer l'économie de marché comme un phénomène universel et atemporel. Ainsi, le modèle de North et Thomas en a irrité plusieurs qui trouvaient aberrant de parler de «prix relatifs» au Moyen-Âge alors que la terre et le travail ne se transigeaient pas sur le marché. D'autres, comme Igartua dans cette revue, ont trouvé l'analyse néo-classique d'Armstrong peu pertinente dans le contexte mercantiliste de la Nouvelle-France ${ }^{9}$.

9. Dans «Le Québec de Pangloss: Structure and Change: an Economic History of Quebec», RHAF, 39,2 (automne 1985): 253-261, Igartua affirmait: «c'est dans la première section, qui porte sur la Nouvelle-France, que le modèle de l'économie de marché s'applique le moins bien: le postulat d'un marché libre, qui sous-tend l'analyse néo-classique, a peu de pertinence dans le contexte mercantiliste des $17^{\mathrm{e}}$ et $18^{\mathrm{e}}$ siècles». Notons qu'une bonne partie de 
Ne confond-on pas la logique économique des «choix rationnels» décrite ci-dessus qui peut s'appliquer dans n'importe quel contexte et le contexte bien particulier qu'est le système économique de marché? La logique économique n'a pas besoin de postuler un marché libre, et «prix relatif» ne signifie pas prix du marché mais bien la valeur réelle déterminée par la rareté relative. Pour prendre un exemple précis, celui de la Grande Peste, tout ce que North et Thomas disaient dans leur jargon d'économiste est qu'elle avait modifié la rareté relative du travail et de la terre. Le travail étant plus rare, son "prix relatif» (sa valeur) augmenta. Ceci entraîna des changements dans le rapport de forces entre seigneurs et vassaux et donc des bouleversements dans le système féodal, le changement institutionnel qu'ils voulaient expliquer $^{10}$.

La question est lancée: peut-être que les économistes voient des homo aconomicus partout, peut-être que les historiens n'en voient nulle part, mais il est difficile de ne pas en voir dans plusieurs travaux d'historiens. Mentionnons deux exemples québécois. Les familles paysannes saguenayennes de Gérard Bouchard ont un comportement tout à fait compatible avec la théorie économique de la famille de Gary Becker dans laquelle la famille et non l'individu maximise son utilité sous contrainte. Encore plus pertinente peut-être, parce que l'utilité de la famille y est maximisée sur une longue période et non de façon statique, est la théorie du cycle de vie qui traite explicitement des relations intergénérationnelles ${ }^{11}$. De même, le marché du logement à Montréal au XVIII ${ }^{\mathrm{e}}$ siècle de la thèse de Daniel Massicotte semble

\footnotetext{
la critique d'Igartua est justifiée mais les faiblesses du volume d'Armstrong proviennent peutêtre plus de l'auteur que de la théorie. Il était beaucoup trop ambitieux d'utiliser la théorie du changement institutionnel de North sans posséder ou effectuer d'études en profondeur des changements institutionnels traités.

10. Peut-être un bel exemple de barrière linguistique interdisciplinaire quoiqu'il faille reconnaitre que North et Thomas expliquent le concept de «prix relatif» presque ad nauseam. Pour vérifier votre propre degré d'allergie, je vous recommande l'article de R. B. Ekelund, R. F. Hébert et R. D. Tollison «The Economics of Sin \& Redemption: Purgatory as a Market Pull Innovation?», Journal of Economic Behavior and Organization, 19,1 (septembre 1992): 1-15. C'est une application de la théorie économique (sans aucune formalisation) pour le moins audacieuse mais intéressante.

11. Voir l'allocution présidentielle de Richard Sutch lors du cinquantenaire de l'Economic History Association en 1990, «All Things Reconsidered: the Life-Cycle Perspective and the Third Task of Economic History», Journal of Economic History, 51,2 (juin 1991): 271-288. Gary Becker vient d'obtenir le Prix Nobel d'économie de 1992 précisément pour sa contribution à l'application de la théorie économique à des sujets traditionnellement non-économiques comme la famille, la criminalité, le mariage. Le niveau de formalisation mathématique de ses travaux est malheureusement relativement élevé.
} 
répondre plus aux impératifs de l'offre et de la demande que le marché du logement actuel tellement contrôlé par l'État ${ }^{12}$.

En terminant, une dernière mise en garde contre la tentation d'égaler théorie néo-classique et concurrence pure et parfaite. Il y a bien longtemps que la théorie économique (en général à un niveau plus avancé) traite d'imperfections de marché. Dans les dernières décennies, les coûts de transactions (créés par l'information imparfaite), le processus politique, le changement institutionnel ont pris la vedette dans ce qu'on appelle encore la «nouvelle économie institutionnelle» dont un des chefs de file est aussi un des chefs de file de la cliométrie, Douglass North.

\section{2 - LA CLIOMÉTRIE, UNE INVENTION AMÉRICAINE}

La cliométrie est née à Williamstown, Massachusetts, en septembre 1957 à la conférence conjointe de l'Economic History Association et du National Bureau of Economic Research. Les heureux parents étaient Alfred Conrad et John Meyer qui y ont présenté leur fameux essai «The Economics of Slavery in the Antebellum South» et un essai méthodologique «Economic Theory, Statistical Inference, and Economic History». Le sujet presque autant que la méthodologie étaient controversés. Soutenant que ce n'est pas parce qu'un système est immoral qu'il est inefficace, ils utilisèrent la théorie économique de l'investissement pour comparer le taux de rendement de la possession d'esclaves à celui de la possession d'autres actifs. Ils en conclurent que l'esclavage était profitable, remettant ainsi en question la vision traditionnelle de l'esclavage sudiste de l'avant-guerre civile comme système économiquement non rentable et non viable, et qui se maintenait seulement parce que l'aristocratie du sud tenait à tout prix à ce mode de $v^{13}{ }^{13}$.

12. Gérard Bouchard et son équipe ont publié plusieurs essais sur le sujet. Celui que j'ai consulté est «Disparités spatiales et modes d'intégration au marché dans l'agriculture québécoise (1921-1951)», texte d'une communication présentée à l'Université de Montréal au colloque sur l'économie rurale des 28-29 février 1992. Quant à Daniel Massicotte, il s'agit de la présentation de sa recherche en cours, «House Characteristics, Location, and Market: Determining Montreal Rental Costs, 1731-1825» à la $18^{\mathrm{e}}$ conférence en histoire économique du Canada, à Vancouver, les 2-3 octobre 1992.

13. Tous deux sont publiés dans le Journal of Political Economy, 66 (avril 1958) et reproduits dans A. H. Conrad et J. R. Meyer, The Economics of Slavery. Assistaient également à la conférence les pères-fondateurs de la cliométrie: Douglass North, William Parker, Robert Gallman, Richard Easterlin et Stanley Lebergott dont les contributions ont fourni les fondements statistiques qui serviront à réécrire la nouvelle histoire économique du XIX $\mathrm{X}^{\mathrm{e}}$ siècle américain. Ces essais ont été publiés dans William Parker, ed., Trends in the American Economy in the Nineteenth Century, National Bureau of Economic Research, Studies in Income and Wealth, vol. 24 (Princeton University Press, 1960). 
Le second enfant de la famille naissait trois ans plus tard à la première conférence officiellement cliométrique à l'Université Purdue, le foyer du mouvement tout au long des années 1960. Robert Fogel y présentait ses mesures des économies externes («social savings») engendrées par le chemin de fer aux États-Unis. Pour ce faire, il comparait la situation réelle en 1890 avec une situation imaginée («counterfactual») sans chemin de fer mais avec des canaux, alors la meilleure alternative. S'il n'y avait pas eu de chemin de fer, le PNB américain en 1890 aurait été de seulement 4,7\% inférieur. Il en concluait donc que le chemin de fer était le mode de transport le plus «efficient» au $\mathrm{XIX}^{\mathrm{e}}$ siècle mais n'était pas, comme le prétendaient plusieurs historiens, indispensable. La communication a soulevé un débat passionné, encore vif dans la mémoire des participants, et l'étude complète publiée en 1965 a eu l'effet d'une bombe. Presque simultanément paraissait celle d'Albert Fishlow qui démontrait que le chemin de fer n'avait pas eu un impact aussi grand qu'on l'avait cru sur l'industrialisation américaine dans la période d'avant-guerre civile. Les travaux de Fogel et de Fishlow ont inspiré un grand nombre de thèses et d'articles qui ont tenté d'évaluer le rôle du chemin de fer dans leurs pays respectifs ${ }^{14}$.

Dans les années 1970, Robert Fogel et Stanley Engerman créaient une controverse encore plus orageuse avec leur analyse économique du système esclavagiste dans laquelle maîtres et esclaves étaient des homo aconomicus. Le débat autour de Time on the Cross (Boston, Little, Brown \& Co, 1974) déborda même du monde académique pour se retrouver dans les médias où des groupes noirs américains accusaient Fogel et Engerman de racisme. Fogel (dont l'épouse est noire) répondait qu'au contraire leur étude réhabilitait le rôle des Noirs dans le développement économique des États-Unis. L'étude fut sévèrement critiquée à l'intérieur du mouvement cliométrique dans P. A. David, H. G. Gutman, R. Sutch, P. Temin, G. Wright, Reckoning with Slavery: a Critical Study in the Quantitative History of American Negro Slavery (Oxford University Press, 1976). Ils accusaient Fogel et Engerman de ne pas reconnaître les limites de l'analyse néo-classique, de ne pas respecter un des critères fondamentaux de la méthode scientifique qui est de présenter ses résultats de manière à être facilement reproduits et de ne pas établir le bien-fondé de ce qu'ils avançaient.

14. R. W. Fogel, Railroads and American Economic Growth; A. Fishlow, American Railroads and the Transformation of the Antebellum Economy (Boston, Harvard University Press, 1965). Pour le Canada par exemple, P. George, «Rates of Return In Railway Investment and Implications for Government Subsidization of the CPR: Some Preliminary Results», Canadian Journal of Economics, 1,4 (1968): 740-762. 
Douglass North est l'autre personnage incontournable des débuts du mouvement, pour ses propres études quantitatives, mais encore plus pour son rôle de missionnaire et de champion de la cliométrie. Il la mit à la portée des étudiants de premier cycle dans Growth and Welfare in the American Past. A New Economic History (Prentice Hall, première édition en 1966, deuxième en 1974, troisième en 1983), une collection d'essais qui démontraient comment la théorie économique la plus élémentaire peut être utile pour contester les interprétations du passé les plus vénérables. Il fut aussi un des principaux inspirateurs de la seconde génération de cliomètres tout en demeurant très critique $^{15}$. Assez rapidement, il plaidera d'ailleurs pour un élargissement de la théorie économique à appliquer à l'étude de l'histoire et passera le reste de sa carrière à tenter d'élaborer une théorie économique du changement institutionnel, des droits de propriété et du rôle de l'État ${ }^{16}$.

À ses débuts dans les années 1960 , on pouvait presque compter sur les doigts des deux mains les membres de la tribu des cliomètres ${ }^{17}$. En une décennie, le mouvement en est venu à dominer totalement l'histoire économique aux États-Unis et la poignée de travaux des

15. Comme le montre cette citation datant de 1965 que le plus anti-cliométrique ne renierait pas: «There has been some valuable work [in the NEH] — particularly in the gathering of statistics - to provide us with some solid footing for analysis, and there have been a few firstrate articles and books, yet the results have been generally disappointing. Too much of it has been dull and unimaginative, and there seems to be a widespread conviction that econometric techniques, the computer, and running a few regressions can substitute for theory and imagination. Some of the new economic history written by economists is of distressingly poor quality. Some of it is so imprecise and fuzzy as to make it difficult, if not impossible, to make any model at all. A good deal of it includes partial-equilibrium analysis of problems with broad general-equilibrium or disequilibrium implications.» «The State of Economic History», American Economic Review, 55 (mai 1965): 90. Les élèves de North qui sont devenus des cliomètres célèbres sont nombreux. Voir R. Ransom, R. Sutch et G. Walton, ed., Explorations in the New Economic History. Essays in Honor of Douglass North (New York, Academic Press, 1982).

16. Il parlera d'élargissement de la théorie dès 1973 dans son allocution présidentielle à l'Economic History Association intitulée «Beyond the New Economic History», Journal of Economic History, 34,1 (mars 1974). Quelques années plus tard, il le répétait pour un auditoire d'économistes: «if economics is a theory of choice subject to specified constraints, a task of economic history is to theorize about those evolving constraints» dans «Structure and Performance: the Task of Economic History», Journal of Economic Literature, 16 (septembre 1978): 963. Pour sa théorie du changement institutionnel, voir les quatre volumes mentionnés dans la note 7.

17. De fait, la fameuse conférence inaugurale de Purdue en 1960 comptait entre 15 et 20 participants selon R. Cameron en entrevue dans la Cliometrics Society Newsletter, 6,1 (février 1991): 6. Mon survol des origines de la cliométrie est loin d'être exhaustif. Par exemple, il y a eu des travaux importants sur les Actes de Navigation par Robert P. Thomas et Gary Walton, le take-off de Rostow par Robert Gallman, le changement technologique par Paul David, l'agriculture sudiste par William Parker et Robert Gallman, les marchés des capitaux par Lance Davis. 


\section{TABLEAU 1}

Affiliation et pays d'origine des membres de la Cliometrics Society

\section{DÉPARTEMENTS}

\begin{tabular}{|c|c|c|c|c|c|c|c|}
\hline & Économie & Histoire & Gestion & $\begin{array}{l}\text { Histoire et } \\
\text { économie }\end{array}$ & Autres & $\begin{array}{c}\text { Non } \\
\text { mentionnés }\end{array}$ & TOTAL \\
\hline États-Unis & 194 & 32 & 24 & 2 & 18 & 62 & 332 \\
\hline Canada & 37 & 3 & 1 & - & - & 2 & 43 \\
\hline \multicolumn{8}{|l|}{ Europe } \\
\hline continentale & 19 & 4 & 1 & 4 & 3 & 22 & 53 \\
\hline Grande-Bretagne & 12 & 4 & 1 & 2 & 2 & - & 21 \\
\hline Japon & 5 & - & - & - & - & - & 5 \\
\hline \multicolumn{8}{|l|}{ Nouvelle-Zélande } \\
\hline et Australie & 3 & - & - & 3 & 3 & - & 9 \\
\hline Autres & 7 & 1 & - & - & - & 4 & 12 \\
\hline TOTAL & 277 & 44 & 27 & 11 & 26 & 90 & 475 \\
\hline
\end{tabular}

Source: The Cliometric Society membership directory, printemps 1992.

années 1960 est devenue un véritable torrent comme le montre le nombre d'études (4 300) répertoriées par McCloskey dans sa Bibliography of Historical Economics to 1980. Un autre bon indicateur de la révolution cliométrique est la proportion des pages du Journal of Economic History qu'occupent les articles cliométriques: elle est passée de 18\% en 1961-1965 à 44\% en 1966-1970 et se situe entre 79 et $83 \%$ depuis $^{18}$. De plus, les cliomètres américains ont leurs rencontres annuelles depuis 1960, leur propre revue Explorations in Economic History et ont formé en 1983 une association The Cliometrics Society qui compte maintenant près de 500 membres à travers le monde. Le tableau 1 présente la compilation (malheureusement partielle, plusieurs membres n'ayant pas indiqué leur affiliation) réalisée à partir du répertoire de 1992 . Comme on pouvait s'y attendre, la grande majorité des membres sont affiliés à des départements nordaméricains d'économie et de gestion.

La situation de l'histoire économique est assez similaire au Canada anglais et aussi, mais dans une moindre mesure, en GrandeBretagne, en Australie et en Nouvelle-Zélande. Depuis peut-être une

18. R. Whaples, «A Quantitative History ...», 293. 
dizaine d'années, il y a une certaine internationalisation en dehors du monde anglo-saxon comme en font foi la formation d'une association européenne d'économie historique et la présence de plus en plus marquée des cliomètres au congrès international d'histoire économique. Nous ne nous attarderons pas sur ce débordement des frontières anglo-saxonnes car ce qui nous intéresse plutôt ici est sa transplantation en sol canadien.

\section{3 - LA CLIOMÉTRIE EN TERRE CANADIENNE}

On peut sans doute situer la naissance de la cliométrie au Canada à octobre 1965, date de la première conférence, tenue à Toronto, sur les méthodes quantitatives en histoire économique du Canada. E. J. Chambers et D. F. Gordon y présentèrent les résultats de leur tentative de mesurer l'impact du staple par excellence, le blé, sur la croissance de l'économie canadienne dans les années du «boom du blé». Ils concluaient qu'au plus $10 \%$ de la croissance du revenu per capita entre 1900 et 1911 pouvait être attribué à la hausse de la demande étrangère pour le blé canadien. Leur procédure et leurs résultats ont été contestés (voir l'item 1 du tableau 3) et la question du «boom du blé» est devenue depuis un staple des congrès canadiens d'histoire économique quantitative.

Il y avait 17 participants à cette première conférence (voir le tableau 2). Il y a maintenant une cinquantaine de cliomètres canadiens. C'est un groupe qui est demeuré informel mais qui en sera au printemps 1994, à l'Université McGill, à sa $19^{\mathrm{e}}$ conférence (qui se tient régulièrement aux 18 mois). Il n'y a pas de revue canadienne d'histoire économique et ses membres publient le plus souvent dans les revues américaines d'histoire économique comme le Journal of Economic History ou Explorations in Economic History ou encore dans les revues canadiennes d'économique comme le Canadian Journal of Economics ou L'Actualité économique.

Il y a bien plus que l'article de John McManus sur les Indiens le seul explicitement relevé dans la revue de Gilles Paquet - dans la contribution des cliomètres à l'histoire économique du Canada. On peut constater l'abondance et la variété de la recherche au tableau 3. Ce tableau qui récapitule les principaux thèmes abordés et les principaux auteurs requiert quelques explications pour éviter tout malentendu. D'abord, il est fort possible que ma perception de ce qu'est la cliométrie ne soit pas nécessairement celle de certains auteurs qui se retrouvent dans ce sommaire. C'est sans doute le cas d'Albert Faucher et de Gilles Paquet qui n'ont jamais voulu porter l'étiquette de cliomètre. Cependant, comme nous l'avons déjà mentionné en note 2 , dans leur article de 1972, «Crise agricole et tensions...», Paquet et 


\section{TABLEAU 2}

Programme de la première conférence de méthodes quantitatives en histoire économique canadienne

\section{[Toronto, 21-22 octobre 1965]}

Hôte: J. Dales (Toronto)

1. E. Chambers et D. Gordon (Washington): «The Staple Theory of Economic Growth: an Empirical Measurement»

2. G. Bertram (Washington): «The Contribution of Education to Economic Growth, 1911-1961: Canada and the United States»

3. A. Green (Queen's): «Regional Aspects of Canada's Economic Growth, 1890-1929»

4. K. Buckley (Saskatchewan): «On the Estimation of National Income in Canada Prior to 1900»

5. M. Urquhart (Queen's): «Historical Statistics: Possibilities and Priorities»

6. E. Safarian (Saskatchewan): «A Programme of Historical Research»

Y participaient également: A. Faucher (Laval), H. Lithwick (Carleton),

D. McDougall (Carleton), M. McInnis (Queen's), R. Olley (Saskatchewan),

J. Rae (UBC), A. Raynauld (Montréal), C. Studness (Federal Reserve Bank of NY), et W. Walsh (Western Ontario).

Source: D. Paterson, «25th Anniversary Meetings - March 1989» dans The Cliometrics Society Newsletter, 4,3 (juillet 1989): 10.

Wallot utilisent explicitement la théorie économique néo-classique. Quant à Faucher, il se définissait souvent comme un historien mais racontait toujours ses histoires dans une perspective et un langage d'économiste. Là réside d'ailleurs, il me semble, toute l'originalité et l'importance de sa contribution à l'historiographie québécoise. Il a fait voir aux historiens les événements majeurs du XIX ${ }^{\mathrm{e}}$ siècle: le rapport Durham, la canalisation, l'Acte d'Union, la Confédération, l'exode des Canadiens français, le développement industriel du Québec et de l'Ontario sous un éclairage d'économiste ${ }^{19}$.

Une étude est considérée cliométrique lorsqu'elle utilise, de façon formalisée ou non, la théorie économique dite néo-classique pour ana-

19. Dix travaux de Gilles Paquet sont inscrits au répertoire de D. McCloskey, A Bibliography of Historical Economics... La majorité des études d'Albert Faucher sont reproduites dans A. Faucher, Histoire économique et unité canadienne (Montréal, Fides, 1970). 


\section{TABLEAU 3}

La cliométrie au Canada des années 1960 à aujourd'hui: un sommaire des principaux thèmes et auteurs

1. Boom du blé

2. Chemins de fer

3. Peuplement de l'Ouest

4. Tarifs

5.a) Agriculture au Québec

5.b) Agriculture en Ontario

6. Industrie manufacturière

7. Commerce des fourrures

8. Monnaie et finances

9. Démographie; travail

10. Statistiques
Chambers \& Gordon (1966); Dales, McManus, Watkins (1967); Bertram (1973); Grant (1974); Lewis (1975).

Faucher (1960); George (1968; 1975); Mercer (1973; 1982); Lewis \& Robinson (1984); Lewis \& MacKinnon (1987); Carlos \& Lewis (1991).

Studness (1964); Norrie (1975; 1980); Marr \& Percy (1978); Southey (1978); Lewis (1981).

Dales (1966); Ankli (1971); Barnett (1976); Harris \& Lewis (miméo).

Paquet \& Wallot (1972); Lewis \& McInnis (1980); McCallum (1980); McInnis (1982); Dupré (1990); Percy \& Szostak (1992).

Pomfret (1976); Marr (1981); McInnis (1992).

Dales (1957); Bertram (1963); McDougall (1971); Armstrong (1979; 1983); Dick (1982); Inwood (1986); Altman (1987); Wylie (1989).

McManus (1972); Carlos (1981; 1982); Carlos \& Nicholas (1990); Carlos \& Lewis (à paraître).

Paterson (1976); Redish (1984; 1990); Bordo \& Redish (1987; 1990); Quigley (1990); Dick \& Floyd (1991).

Faucher (1964); Green \& Green (1993); Green \& MacKinnon (1988); MacKinnon (1990);

Raynauld (1961); Bertram (1964); Urquhart \& Buckley (1965, 1983); Green (1971); Green (1986); McInnis (1986); Urquhart (1986); Dupré (1988).

Sources: voir annexe. 
lyser un phénomène historique. Ce critère correspond à la définition que j'ai défendue dans la section 1 et est celui que préfère également Whaples, l'auteur de la plus récente compilation des travaux cliométriques aux États-Unis ${ }^{20}$. J'y ai ajouté les travaux statistiques de base des Urquhart, Green, Bertram et Raynauld, qui ont été, et sont toujours pour la plupart, des piliers du groupe canadien de cliométrie.

Finalement, ce sommaire n'a pas la prétention d'être exhaustif. Il veut seulement illustrer ce qu'ont pu être les réalisations de la cliométrie canadienne. Plusieurs cliomètres canadiens tels Michael Bordo, Knick Harley, Michael Huberman, Mary MacKinnon - et j'en oublie - ont effectué une grande partie de leur recherche sur des sujets non canadiens qui ne sont pas inclus dans ce tableau. De même, certains autres, comme Ian Drummond, H. V. Nelles et Christopher Armstrong, se retrouvent exclus parce qu'ils ont abordé d'autres thèmes que ceux présentés ici.

Au Canada comme aux États-Unis, de nombreux mythes ont été remis en question ou au moins examinés de plus près. Le boom du blé par Chambers et Gordon, le tarif de 1879 par John Dales et par Richard Harris et Frank Lewis, la rentabilité sociale du Canadien Pacifique par Peter George, l'inefficacité des paysans canadiensfrançais au XIX ${ }^{\mathrm{e}}$ siècle par Frank Lewis et Marvin McInnis, le commerce des fourrures de la Baie d'Hudson par Ann Carlos, la Révolution tranquille, ou du moins son côté finances publiques, par Ruth Dupré, ne sont que quelques exemples.

\section{4 - LA FIN DE LA GUERRE FROIDE ENTRE CLIOMĖTRES ET HISTORIENS}

C'est peut-être Peter McClelland dans son excellent essai méthodologique de 1975 qui résume le mieux les relations qui ont prévalu pendant presque deux décennies entre cliomètres et historiens:

Two warring camps confront each other with distrust, occasional hostility, and a minimum of communication. On the one side is a small band of competent economists: rigorous in their methods, preoccupied with generalization, and accused of battering the subtle fabric of history into the Procrustean bed dictated by their theoretical models, available data, and statistical techniques. On

20. Comme il n'y a pas de définition précise de ce qu'est un article cliométrique, Whaples en utilise trois pour construire son indicateur de la révolution cliométrique aux ÉtatsUnis (le pourcentage de pages dans le Journal of Economic History). Celle qu'il préfere est sa deuxième qui comprend les articles qui font usage de la théorie économique avec ou sans méthodes quantitatives. Voir R. Whaples, «A Quantitative History...», 294. 
the other side is ranged the vast majority of historians, their preferences and scholarship reflecting the antiquarian's instinct for the unique and the humanist's distrust of universal propositions. Their faults - or so the other side maintains - include a propensity to condemn cliometrics with little understanding of its methods... ${ }^{21}$ »

Le climat des relations entre les deux groupes s'est beaucoup adouci depuis une dizaine d'années. Il y a plusieurs indicateurs de ce rapprochement. Le livre de deux illustres représentants de chaque camp, R. W. Fogel et de G. R. Elton, Which Road to the Past? Two Views of History (Yale University Press, 1983) en est un important. Plus significatif encore pour les historiens québécois est le tout dernier volume d'histoire économique du Canada enfin écrit en collaboration par un économiste et un historien, K. Norrie et D. Owram, A History of the Canadian Economy (Harcourt Brace Jovanovitch, 1991). Un passage de leur préface vaut la peine d'être cité ici au long:

Historians and economists both have, through the years written and taught in the field while eyeing their rivals, their demeanours part supportive and enthusiastic, part suspicious and critical. The result has been two streams of literature that often been surprisingly divergent in method and readership. This fact seems to rob the field of its greatest strengths - its multidisciplinary nature. The coauthors hope to bring the strengths of the two disciplines to reinforce each other and act as a check on the excessively extravagant claims of each.

Voilà de quoi être très optimiste quant à l'avenir du ménage à trois. Je ne peux ni ne veux dénier toutes les difficultés que rencontre l'économiste-historien «cette espèce hybride exposée à l'opprobre» comme le disait si bien Albert Faucher. C'est beaucoup demander à une même personne d'avoir de la profondeur historique, de faire du bon travail de recherche en archives, de maîtriser la théorie économique et les méthodes d'analyse statistique appropriées. Je ne crois pas cependant que cela explique le peu d'enthousiasme des économistes québécois pour l'histoire économique. C'est plutôt que la marginalité est une entreprise fort risquée. Bien sûr si l'on trouve sa niche, les bénéfices d'être marginal sont très intéressants (les économistes parleraient d'une rente de rareté) mais les probabilités que cela arrive sont faibles. Alors, pour un étudiant de doctorat en économie ou un professeur en début de carrière, le coût de s'engager explicitement et complètement dans la voie de l'histoire économique est énorme. Au

21. P. D. McClelland, Causal Explanation and Model Building.., 243. 
Québec, la marginalité est particulièrement forte parce qu'il y bien peu de tradition d'histoire économique dans les départements d'économique: il n'y a jamais eu plus d'un ou deux économistes-historiens par génération.

Cependant, il existe un groupe fort dynamique de cliomètres, ces économistes intéressés à l'histoire économique, aux États-Unis et un au Canada qui, toutes proportions gardées, n'a rien à envier à son voisin américain. Plusieurs d'entre eux présentent les résultats de leurs recherches de façon tout à fait compréhensible pour des nonéconomistes. Entre eux et les historiens québécois qui se préoccupent de questions d'ordre économique, la communication doit s'établir.

\section{ANNEXE \\ Références bibliographiques complètes du tableau 3}

Liste des abréviations utilisées
$\mathrm{AE} \quad$ : Actualité économique
CJE : Canadian Journal of Economics
CJEPS : Canadian Journal of Economics and Political Science
EEH : Explorations in Economic History
JCS : Journal of Canadian Studies
JEH : Journal of Economic History
JME : Journal of Monetary Economics
RHAF : Revue d'histoire de l'Amérique française
RS : Recherches sociographiques

ALTMAN, M., «A Revision of Canadian Economic Growth: 1870-1910...», CJE (février 1987).

ANKLI, R. E., «The 1854 Reciprocity Treaty», CJE (février 1971).

ARMSTRONG, R., «L.'industrie de l'amiante au Québec, 1878-1929», RHAF (septembre 1979); «Le développement des droits miniers au Québec à la fin du XIX ${ }^{\mathrm{e}}$ siècle», $A E$ (septembre 1983).

BARNETT, D. F., «The Galt Tariff: Incidental or Effective Protection», CJE (août 1976).

BERTRAM, G. W., «Economic Growth and Canadian Industry, 18701915...», CJEPS (mai 1963); «Historical Statistics on Growth and Structure of Manufacturing in Canada 1870-1957», Canadian Political Science Association Conferences on Statistics 1962 and 1963 (Toronto 1964); "The Relevance of the Wheat Boom in Canadian Economic Growth», CJE (novembre 1973). 
BORDO, M.D. et REDISH, A., «Why did the Bank of Canada Emerge in 1935?», JEH (juin 1987); «Credible Commitment and Exchange Rate Stability: Canada's Interwar Experience», CJE (mai 1990).

CARLOS, A., «The Causes and Origins of the North American Fur Trade Rivalry: 1804-1810», JEH (décembre 1981); «The Birth and Death of Predatory Competition in the North American Fur Trade: 1810-1821», EEH (avril 1982); avec S. NICHOLAS, «Agency Problems in Early Chartered Companies: the Case of the Hudson's Bay Company», JEH (décembre 1990); avec F. Lewis, «The Profitability of Early Canadian Railroads...», Strategic Factors in Nineteenth Century American Economic History. A Volume to Honor R.W. Fogel (NBER et University of Chicago Press, 1991); avec F. LEWIS, «Depletion in the Lands of the Hudson's Bay Company, 1700-1770», à paraître JEH.

CHAMBERS, E. J. et GORDON, D. F., «Primary Products and Economic Growth: an Empirical Measurement», JPE (août 1966).

DALES, J. H., Hydroelectricity and Industrial Development in Quebec, 1898-1940 (HarvardUniversity Press, 1957); The Protective Tariff in Canada's Development (University of Toronto Press, 1966); avec J. C. McMANUS et M. H. WATKINS, «Primary Products and Economic Growth: a Comment», JPE (décembre 1967).

DICK, T. J. O., «Canadian Newsprint, 1913-1930: National Policies and the North American Economy», JEH (septembre 1982); avec J. E. FLOYD, «Balance of Payments Adjustment Under the International Gold Standard: Canada, 1871-1913», EEH (avril 1991).

DUPRÉ, R.; «Un siècle de finances publiques québécoises: 1867-1969», $A E$ (décembre 1988); «Regulating the Quebec Dairy Industry, 1905-1921: Peeling Off the Joseph Label», JEH (juin 1990).

FAUCHER, A., «Some Aspects of the Financial Difficulties of the Province of Quebec», CJEPS (novembre 1960); «L'émigration des Canadiens français: position du problème et perspective», $R S$ (sept.-déc. 1964).

GEORGE, P. J., «The Rates of Return in Railway Investment and Implications for Government Subsidization of the CPR: Some Preliminary Results», CJE (novembre 1968); et «The Rates of Return in Railway Investment and Implications for Government Subsidization of the CPR: Some Further Remarks», CJE (novembre 1975).

GRANT, D., "The Staple Theory and its Empirical Measurement», JPE (décembre 1974).

GREEN, A. G., Regional Aspects of Canadian Economic Growth (University of Toronto Press, 1971); avec M. MacKINNON, «Unemployment and Relief in Canada During the Great Depression», Interwar Unemployment 
in International Perspective (London, 1988); «Growth and Productivity Change in the Canadian Railway Sector 1871-1926», Long-Term Factors in American Economic Growth. NBER Studies in Income and Wealth vol. 51 (University of Chicago Press, 1986); avec D. A. GREEN, «Balanced Growth and the Geographical Distribution of European Immigrant Arrivals to Canada, 1900-1912», EEH (janvier 1993).

HARRIS, R. et LEWIS, F., «The Impact of the National Policy on Canadian Manufacturing, 1880 to 1890 » (mimeo, Queen's University).

INWOOD, K. E., The Canadian Charcoal Iron Industry (Garland Publ. Co, 1986).

LEWIS, F., «The Canadian Wheat Boom and Per Capita Income, New Estimates», JPE (décembre 1975); avec M. McINNIS, «The Efficiency of the French-Canadian Farmer in the Nineteenth Century», JEH (septembre 1980); «Farm Settlement on the Canadian Prairies, 1898 to 1911», JEH (septembre 1981); avec D. R. ROBINSON, «The Timing of Railway Construction on the Canadian Prairies», CJE (mai 1984); avec M. McKINNON, «Grovernment Loan Guarantees and the Failure of the Canadian Northern Railway», JEH (mars 1987).

MacKINNON, M., «Relief not Insurance: Canadian Unemployment Relief in the 1930s», EEH (janvier 1990).

MARR, W., «The Wheat Economy in Reverse: Ontario's Wheat Production 1887-1917», CJE(février 1981); avec M. PERCY, «The Government and the Rate of Prairie Settlement», CJE (novembre 1978).

McCALlUM, J., Unequal Beginnings: Agriculture and Economic Development in Quebec and Ontario until 1870 (University of Toronto Press, 1980).

McDOUGALL, D. M., «Canadian Manufactured Commodity Output, 18701915», CJE (février 1971).

McINNIS, R. M., «A Reconsideration of the State of Agriculture in Lower Canada...», Canadian Papers in Rural History, vol. 3 (1982); «Output and Productivity in Canadian Agriculture 1870 to 1926», Long-Term Factors in American Economic Growth. NBER Studies in Income and Wealth, vol. 51 (University of Chicago Press, 1986); Perspectives on Ontario Agriculture 1815-1930 (Langdale Press, 1992).

McMANUS, J., «An Economic Analysis of Indian Behaviour in the North American Fur Trade», JEH (mars 1972).

MERCER, L. J, «Rates of Return and Government Subsidization of the CPR: an Alternative View», CJE (août 1973); Railroads and Land Grant Policy (Academic Press, 1982). 
NORRIE, K. H., «The Rate of Settlement of the Canadian Prairies 18701911», JEH (juin 1975); «Cultivation Techniques as a Response to Risk in Early Canadian Prairie Agriculture», EEH (octobre 1980).

PAQUET, G. et WALLOT, J.-P., «Crise agricole et tensions socio-ethniques dans le Bas-Canada, 1802-1812...», RHAF (septembre 1972).

PATERSON, D. G., British Direct Investment in Canada, 1890-1914 (University of Toronto Press 1976).

PERCY, M. B. et SZOSTAK, R., «The Political Economy of the Abolition of Seigneurial Tenure in Canada East», EEH (janvier 1992).

POMFRET, R. W. T., «The Mechanization of Reaping in 19th Century Ontario», JEH (juin 1976).

QUIGLEY, N. C., «Discrimination in Bank Lending Policies: a Test Using Data from The Bank of Nova Scotia, 1900-1934», CJE (1990).

RAYNAULD, A., Croissance et structure économique de la Province de Québec (Gouvernement du Québec 1961).

REDISH, A., «Why Was Specie Scarce in Colonial Economies? An Analysis of the Canadian Currency, 1796-1830», JEH (1984); «Monetary Lessons from Canada: a Review Essay», JME (décembre 1990).

SOUTHEY, C., «The Staples Thesis, Common Property and Homesteading», CJE (août 1978).

STUDNESS, C. M., «Economic Opportunity and the Westward Migration of Canadians During the Late Nineteenth Century», CJEPS (novembre 1964).

URQUHART, M. C., «New Estimates of G.N.P., Canada, 1870-1926...», Long-Term Factors in American Economic Growth, NBER Studies in Income and Wealth, vol. 51 (University of Chiçago Press, 1986); avec BUCKLEY, K. A. H., eds., Historical Statistics of Canada (Macmillan 1965 et Statistics Canada 1983).

WYLIE, P., «Technological Adaptation in Canadian Manufacturing, 19001929», JEH (septembre 1989). 